\title{
Disks around FUor-type young eruptive stars with ALMA
}

\author{
Fernando Cruz-Sáenz de Miera ${ }^{1}$, Ágnes Kóspál ${ }^{1,2}$, Péter Ábraham¹, \\ Hauyu Baobab $\mathrm{Liu}^{3}$ and Michihiro Takami ${ }^{4}$ \\ ${ }^{1}$ Konkoly Observatory, Budapest, Hungary \\ email: cruzsaenz.fernando@csfk.mta.hu \\ ${ }^{2}$ MPIA, Heidelberg, Germany; ${ }^{3}$ ESO, Garching, Germany; ${ }^{4}$ ASIAA, Taipei, Taiwan
}

\begin{abstract}
A long-standing problem of the general paradigm of low-mass star formation is the "luminosity problem": protostars are less luminous than theoretically predicted. One possible solution is that the accretion process is episodic. FU Orionis-type stars (FUors) are thought to be the visible examples for objects in the high accretion state and it is still debated what physical mechanism triggers the phenomenon. For many of these objects their disk properties are still largely unknown so we conducted a deep, high spatial resolution (down to 20 au) ALMA Band 6 $(1.3 \mathrm{~mm})$ dust continuum survey of a sub-sample of known FUors. Here we present preliminary results of our survey, including the mass, size and spectral slope of each disk.
\end{abstract}

Keywords. stars: pre-main-sequence, stars: circumstellar matter, techniques: interferometric

\section{Introduction}

Young eruptive stars are low-mass pre-main sequence stars showing large outbursts in visible light $(\Delta \mathrm{V} \sim 5)$. See Audard et al. (2014) for a review of these objects. The cause for these events is thought to be a temporary increase of accretion rate going from $10^{-8}$ to $10^{-4} \mathrm{M}_{\odot}$ year $^{-1}$. The duration of these outbursts is $\sim 100 \mathrm{~s}$ years; therefore, they contribute significantly to the final stellar mass. The outbursts clear the material surrounding the star, helping the transition from the embedded Class I to disk-only Class II. Recent studies have shown that FUor disks are smaller and more massive than previously thought (Cieza et al. 2018, and Liu et al. 2018).

\section{Observations \& Analysis}

ALMA Band 6 with two continuum windows plus ${ }^{12} \mathrm{CO},{ }^{13} \mathrm{CO}$ and $\mathrm{C}^{18} \mathrm{O}$ using the $7 \mathrm{~m}$ and $12 \mathrm{~m}$ arrays, with baselines from $\sim 9 \mathrm{~m}$ to $\sim 3600 \mathrm{~m}$. Here we present only the continuum results. Self-calibration of phases and amplitudes for targets with high enough SNR. Measured radii, inclination and position angles by fitting a 2D Gaussian model convolved with the synthesized beam. Estimated disk masses assuming 100:1 gas-to-dust ratio, $\kappa=2.2 \mathrm{~cm}^{2} \mathrm{~g}^{-1}$ and $\mathrm{T}_{\text {dust }}=50 \mathrm{~K}$. The $\alpha$ spectral index was calculated using the two continuum windows.

\section{Results}

The L1551 IRS 5 image shows the two circumstellar disks if the system, N and S, separated by $51 \mathrm{au}$, and a resolved circumbinary ring (CBR), extending from $\sim 100$ to $\sim 200$ au. The circumstellar disks have radii of 9.4 au and 6.9 au for $\mathrm{N}$ and $\mathrm{S}$, respectively. $\mathrm{N}, \mathrm{S}$ and the CBR have inclinations of 47,39 and $45^{\circ}$, and PAs of 148,150 and $150^{\circ}$, 
respectively, i.e. they are aligned, as previously determined by Lim et al. (2016). N shows extension towards the north which appears to connect it to the CBR. The CBR has a spectral index $\alpha=3.3 ; \mathrm{N}$ and $\mathrm{S}$ have $\alpha \approx 2$; the rest of the sample has typical $\alpha$ value between 2.2 and 3.8, with the exception of $\mathrm{AR} 6 \mathrm{~B}$ with $\alpha \approx 1.4$. The other FUors have disks ranging from 20 to $50 \mathrm{au}$ with the exception of $\mathrm{AR} 6 \mathrm{~B}$ with a radius of $70 \mathrm{au}$. The masses of all the circumstellar disks in our sample range from $10^{-4}$ to $10^{-3} \mathrm{M}_{\odot}$.

\section{Conclusions}

AR 6B, Bran 76, OO Ser and V899 Mon show dust extensions at a $\sim 10 \sigma$ level. Haro 5a shows a faint companion 0.7" to the southwest. V346 Nor shows a much larger and fainter envelope. The values we report are 2-3 orders of magnitude below the values reported by Cieza et al. (2018); however, because of high optical depths and the uncertainty on the dust parameters, our mass estimates should be taken as lower limits. Difference of spectral index between circumbinary ring and circumstellar disks in L1551 IRS 5 can be an indication of grain growth or of high optical depth in the circumstellar disks. The other FUor disks show a wide range of values for the spectral index without a clear trend. A more detailed analysis of L1551 IRS 5 will be presented in Cruz-Sáenz de Miera et al. (in preparation) and for the full sample in Kóspál et al. (in preparation). This project has received funding from the European Research Council (ERC) under the European Union's Horizon 2020 research and innovation programme under grant agreement No 716155 (SACCRED, PI:Á. Kóspál).

\section{References}

Audard, M., Ábrahám, P., Dunham, M. M., Green, J. D., Grosso, N., Hamaguchi, K., Kastner, J. H., Kóspál, Á., Lodato, G., Romanova, M. M., Skinner, S. L., Vorobyov, E. I. and Zhu, Z. 2005, Protostars and Planets VI, pp. 387-410

Cieza, L. A., Ruíz-Rodríguez, D., Perez, S., Casassus, S., Williams, J. P., Zurlo, A., Principe, D. A., Hales, A., Prieto, J. L., Tobin, J. J., Zhu, Z. and Marino, S. 2018, MNRAS, 474, 4347

Lim, J., Yeung, P. K. H., Hanawa, T., Takakuwa, S., Matsumoto, T. and Saigo, K. 2016, ApJ, 826,153

Liu, H. B., Dunham, M. M., Pascucci, I., Bourke, T. L., Hirano, N., Longmore, S., Andrews, S., Carrasco-González, C., Forbrich, J., Galván-Madrid, R., Girart, J. M., Green, J. D., Juárez, C., Kóspál, Á., Manara, C. F., Palau, A., Takami, M., Testi, L. and Vorobyov, E. I. 2018, A\&A, 612, A54
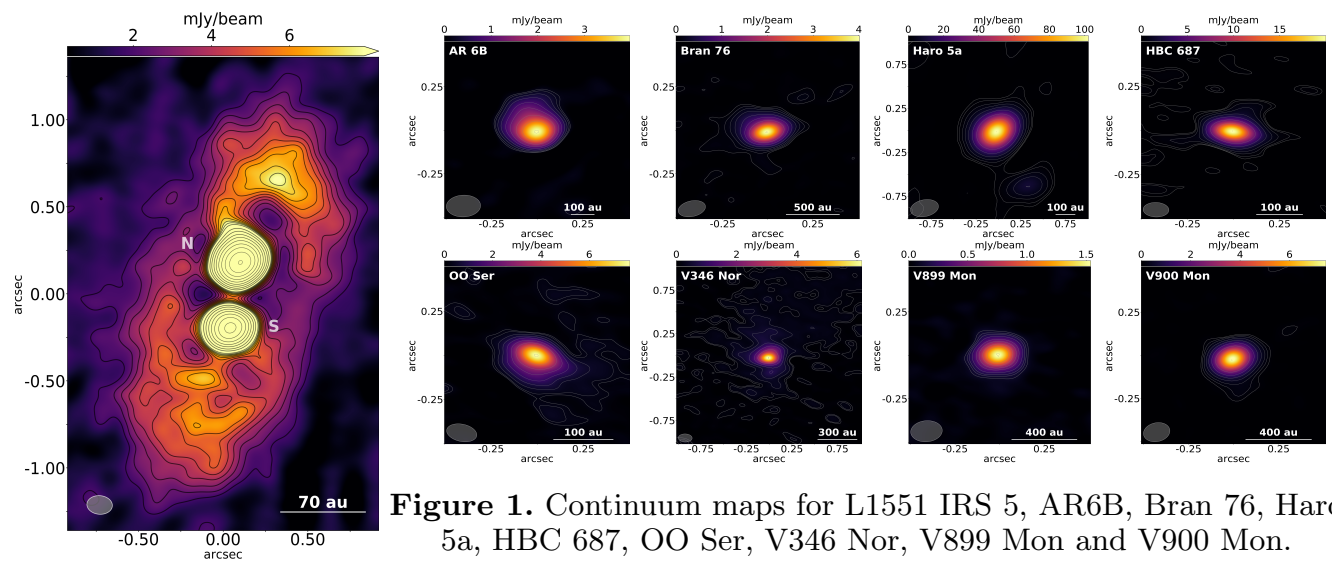

Figure 1. Continuum maps for L1551 IRS 5, AR6B, Bran 76, Haro 5a, HBC 687, OO Ser, V346 Nor, V899 Mon and V900 Mon. 